Check for updates

Cite this: J. Mater. Chem. A, 2021, 9, 19725

\title{
Three-in-one: achieving a robust and effective hydrogen-evolving hybrid material by integrating polyoxometalate, a photo-responsive metal- organic framework, and in situ generated Pt nanoparticles $\dagger$
}

\begin{abstract}
Le Jiao, Yuanyuan Dong, Xing Xin, Ruijie Wang and Hongjin Lv (D)*
A Wells-Dawson-type polyoxometalate $\left(\left[\mathrm{P}_{2} \mathrm{~W}_{18} \mathrm{O}_{62}\right]^{6-}\right.$, denoted as $\left.\mathrm{P}_{2} \mathrm{~W}_{18}\right)$, a photo-responsive $\mathrm{Zr}$-based metal-organic framework (MOF, NU-1000), and in situ generated Pt nanoparticles were successfully integrated into a three-in-one hybrid material $\left(\mathrm{P}_{2} \mathrm{~W}_{18}(\mathrm{aNU}-1000-\mathrm{Pt})\right.$ using a facile impregnation and subsequent photoreduction method. The resulting three-in-one hybrid material exhibits effective and robust activity towards photocatalytic hydrogen production in a water-compatible system under Xelamp irradiation, achieving a hydrogen production of $35100 \mu \mathrm{mol} \mathrm{g}{ }^{-1}$ and a turnover number (TON) of 5484 versus moles of $\mathrm{Pt}$ after 5 days of reaction. The post-catalysis three-in-one hybrid composite could be easily recycled at least 5 times without declining catalytic activity. Moreover, such a watercompatible photocatalytic system also reveals potential practical applications for catalyzing hydrogen production under natural sunlight irradiation. A possible photocatalytic mechanism was elucidated based on various photophysical and spectroscopic analyses, proving the importance of synergistic cooperation of the NU-1000 MOF, the $\mathrm{P}_{2} \mathrm{~W}_{18}$ cluster, and in situ generated Pt NPs in such a three-in-one $\mathrm{P}_{2} \mathrm{~W}_{18} @ \mathrm{NU}-1000-\mathrm{Pt}$ hybrid photocatalyst.
\end{abstract}

Received 2nd April 2021

Accepted 25th May 2021

DOI: $10.1039 / \mathrm{d} 1 \mathrm{ta0} 2792 \mathrm{a}$

rsc.li/materials-a

\section{Introduction}

Energy shortages and environmental pollution caused by fossil fuels are currently a major challenge. Solar energy, as a clean and sustainable energy source, is a very promising alternative solution to the increasingly serious energy crisis. The conversion of solar energy into storable chemical energy via photocatalytic water splitting for hydrogen generation has therefore received widespread attention. Various materials, such as carbon-based materials, ${ }^{\mathbf{1 - 4}}$ semiconductors, ${ }^{5,6}$ conjugated polymers, ${ }^{7,8}$ covalent organic frameworks, ${ }^{9,10}$ noble metal nanoparticles, ${ }^{\mathbf{1 1 , 1 2}}$ metal organic frameworks, ${ }^{\mathbf{1 3 , 1 4}}$ polyoxometalates, ${ }^{15,16}$ etc., have been investigated for photocatalytic hydrogen production. Polyoxometalates (POMs), as an emerging type of multi-electron-transfer catalyst, have been employed as photocatalysts for water splitting due to their highly tunable physicochemical properties, versatile synthetic methodology and multi-electron-transfer ability. With the assistance of soluble sensitizers such as $\left[\mathrm{Ru}(\mathrm{bpy})_{3}\right]^{2+}$ and

MOE Key Laboratory of Cluster Science, School of Chemistry and Chemical Engineering, Beijing Institute of Technology, Beijing 102488, P. R. China. E-mail: hlv@bit.edu.cn

$\dagger$ Electronic supplementary information (ESI) available. See DOI: $10.1039 / \mathrm{d} 1 \mathrm{ta} 02792 \mathrm{a}$
$\left[\operatorname{Ir}(\text { ppy })_{2}(\text { bpy })\right]^{+}$, POMs could exhibit outstanding photocatalytic activity. ${ }^{17-21}$ In spite of their high TON for hydrogen generation in a homogeneous photocatalytic system, the recycling of POMs is still an urgent problem to be solved. To improve the recyclability of POM photocatalysts, a series of support materials, such as graphene, ${ }^{22}$ carbon nanotubes, ${ }^{23}$ metal oxides, ${ }^{24}$ zeolites, ${ }^{25}$ porous aromatic frameworks, ${ }^{26}$ covalent organic frameworks, ${ }^{27}$ and metal organic frameworks (MOFs) ${ }^{28}$ have been employed to construct POM-based composites for heterogeneous photocatalytic systems.

MOFs, a class of materials composed of metal ions/clusters and organic linkers, have attracted widespread attention for immobilizing POMs due to their porous structures, rich chemical functionality, high surface area, crystallinity and chemical stability. ${ }^{29-35}$ The resulting POM@MOF composites are thus widely utilized in the photocatalytic hydrogen production system..$^{36,37}$ For example, Lin and co-workers integrated a Wells-Dawson-type POM $\left[\mathrm{P}_{2} \mathrm{~W}_{18} \mathrm{O}_{62}\right]^{6-}$ into a $\mathrm{Zr}$-based MOF bearing the $\left[\mathrm{Ru}(\mathrm{bpy})_{x}\right]^{2+}$ moiety, and encapsulated a cobalt-containing POM $\left(\mathbf{P}_{\mathbf{2}} \mathbf{W}_{\mathbf{1 7}} \mathbf{C o}\right)$ and photosensitizer into the pores of MIL-101 to achieve hydrogen production under visible light irradiation, respectively. ${ }^{38,39} \mathrm{Du}$ et al. incorporated a high-nuclear $\left\{\mathrm{Cu} 24 \mathrm{I}\left(\mu_{3}-\mathrm{Cl}\right)_{8}\left(\mu_{4}-\mathrm{Cl}\right)_{6}\right\}$-based POM into ZZULI-1 to construct a dual-functionalized photocatalyst for visiblelight-driven $\mathrm{H}_{2}$ and $\mathrm{O}_{2}$ evolution. ${ }^{40}$ These applications in 
photocatalytic hydrogen production demonstrate the potential value and diversity of POM@MOF composites. It is worth mentioning that the long-term stability and reusability of these POM@MOF composites are largely determined by the size match between the POM guest molecule and MOF host as well as the physicochemical properties of MOFs. Among various MOF supports, NU-1000, a Zr-based MOF with a prominent photo-response and diverse aperture size (mesopore and micropore), exhibits outstanding physicochemical stability and therefore has been widely employed as the host of composite photocatalysts. For instance, to improve the photogenerated charge separation efficiency, BiOI nanoparticles and CdSe nanocrystals have been successfully incorporated into NU-1000, respectively, and both hybrid composites exhibited excellent photocatalytic activity. ${ }^{\mathbf{4 1 - 4 3}}$ To enhance the photocatalytic stability, Keggin-type $\mathrm{H}_{5} \mathrm{PV}_{2} \mathrm{Mo}_{10} \mathrm{O}_{40}$ and $\mathrm{H}_{3} \mathrm{PW}_{12} \mathrm{O}_{40}$ have also been immobilized into $\mathrm{NU}-1000$ and the resulting hybrid materials showed efficient catalytic activities for aerobic oxidation of a mustard gas simulant, 2-chloroethyl ethyl sulfide. ${ }^{\mathbf{4 4 , 4 5}}$ However, it is rarely reported about the size matching issue for the immobilization of a POM into NU-1000, ${ }^{37}$ especially in a solar-driven hydrogen generation system.

Herein, a classical Wells-Dawson-type POM $\mathbf{P}_{\mathbf{2}} \mathbf{W}_{\mathbf{1 8}}$ is therefore immobilized into NU-1000 to construct the $\mathbf{P}_{\mathbf{2}} \mathbf{W}_{\mathbf{1 8}} @ \mathrm{NU}$ 1000 composite given the suitable match in size (Scheme 1). We further fabricate a three-in-one $\mathbf{P}_{\mathbf{2}} \mathbf{W}_{\mathbf{1 8}} @ N U-1000-\mathrm{Pt}$ hybrid photocatalyst through the in situ formation of Pt nanoparticles (Pt NPs) and utilize it for the photocatalytic hydrogen evolution reaction. As a co-catalyst, Pt NPs have been investigated for photocatalytic hydrogen evolution, ${ }^{46-52}$ including Pt-POM and Pt-MOF composites. ${ }^{53-65}$ These studies reveal that the photocatalytic efficiency is largely associated with the Pt location relative to the POM and/or MOF. In our work, the in situ generated Pt NPs could be immobilized into the pores of the NU-1000 host or supported on the surface of the $\mathbf{P}_{\mathbf{2}} \mathbf{W}_{\mathbf{1 8}} @ \mathrm{NU}$ 1000 composite to generate the three-in-one hybrid photocatalyst, which could not only prevent the leaching out of $\mathbf{P}_{\mathbf{2}} \mathbf{W}_{\mathbf{1 8}}$ guest molecules, but also promotes photogenerated charge separation efficiency to improve the photocatalytic performance. The resulting three-in-one $\mathbf{P}_{\mathbf{2}} \mathbf{W}_{\mathbf{1 8}} @$ @U-1000-Pt photocatalyst therefore exhibits effective and robust photocatalytic activity with a hydrogen production of $6023 \mu \mathrm{mol} \mathrm{g}{ }^{-1}$ after $12 \mathrm{~h}$

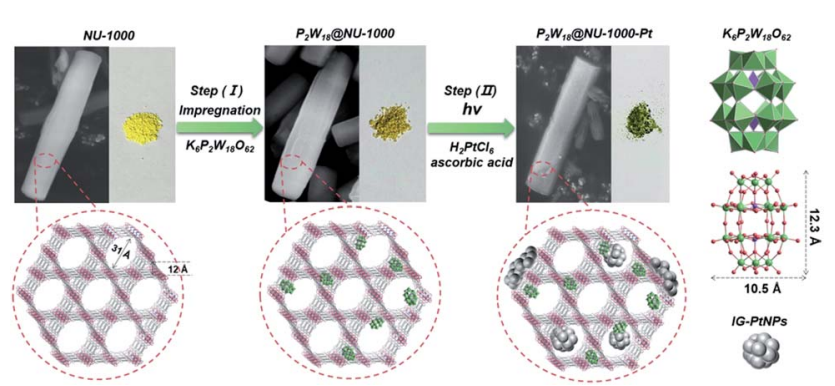

Scheme 1 Schematic illustration of the preparation route of the threein-one $\mathrm{P}_{2} \mathrm{~W}_{18} @ \mathrm{NU}-1000-\mathrm{Pt}$ hybrid composite. The microscopic illustration of the structure is shown in the red circles. illumination that continuously increases to $35100 \mu \mathrm{mol} \mathrm{g}^{-1}$ after 5 days of reaction.

\section{Results and discussion}

The three-in-one $\mathbf{P}_{\mathbf{2}} \mathbf{W}_{\mathbf{1 8}} @ N U-1000-\mathrm{Pt}$ hybrid composite was prepared via a facile impregnation (Step (I)) and subsequent photoreduction (Step (II)) approach as illustrated in Scheme 1. The NU-1000 (yellow powder) was added into the $\mathbf{P}_{\mathbf{2}} \mathbf{W}_{\mathbf{1 8}}$ aqueous solution, and then the mixture was subjected to ultrasonication, stirring, and post-treatment to obtain the dark yellow $\mathbf{P}_{\mathbf{2}} \mathbf{W}_{\mathbf{1 8}} @ \mathrm{NU}-1000$ powder as described in the Experimental section. Subsequently, during the photocatalytic reaction (Step (II)), the NU-1000 host was photoexcited to generate electron-hole pairs where the photogenerated electrons could be transferred to $\mathbf{P}_{2} \mathbf{W}_{\mathbf{1 8}}$ and the $\mathrm{H}_{2} \mathrm{PtCl}_{6}$ to in situ form the $\mathrm{Pt}$ NPs, obtaining the three-in-one $\mathbf{P}_{\mathbf{2}} \mathbf{W}_{\mathbf{1 8}} @ \mathrm{NU}-1000-\mathrm{Pt}$ hybrid composite (dark green powder in Scheme 1). The detailed preparation procedures are described in the Experimental section. Inductively coupled plasma-atomic emission spectrometry (ICP-AES) was employed to determine the loading amount of $\mathbf{P}_{\mathbf{2}} \mathbf{W}_{\mathbf{1 8}}$ in terms of $\mathbf{P}_{\mathbf{2}} \mathbf{W}_{\mathbf{1 8}}$ per $\left\{\mathrm{Zr}_{6}\right\}$ node in the $\mathbf{P}_{\mathbf{2}} \mathbf{W}_{\mathbf{1 8}} @$ NU-1000 composite (Table 1). The optimal $\mathbf{P}_{\mathbf{2}} \mathbf{W}_{\mathbf{1 8}} /\left\{\mathrm{Zr}_{6}\right\}$ ratio was found as 0.34 for the photocatalytic hydrogen evolution reaction, and 0.34- $\mathbf{P}_{\mathbf{2}} \mathbf{W}_{\mathbf{1 8}} @ \mathrm{NU}-1000$ was therefore selected as the representative sample for a series of characterization experiments unless otherwise noted. Scanning Electron Microscopy (SEM) images illustrate that the morphology of 0.34$\mathbf{P}_{2} \mathbf{W}_{18} @ N U-1000$ still maintains a uniform rod-like structure as that of NU-1000 (Fig. 1a and $\mathrm{S} 1 \dagger$ ). The impregnation of the $\mathbf{P}_{2} \mathbf{W}_{18}$ guest does not change the smooth surface and morphology of the NU-1000 host. The similar Powder X-ray Diffraction (PXRD) patterns of NU-1000 and 0.34- $\mathbf{P}_{\mathbf{2}} \mathbf{W}_{\mathbf{1 8}} @ \mathrm{NU}$ 1000 also prove that the immobilization of $\mathbf{P}_{\mathbf{2}} \mathbf{W}_{\mathbf{1 8}}$ has a negligible effect on the geometrical structure of the NU-1000 host (Fig. S2 $\dagger$ ). But the absolute intensity of the diffraction signals at the small angles decreases after $\mathbf{P}_{\mathbf{2}} \mathbf{W}_{\mathbf{1 8}}$ immobilization, which to some extent proves the incorporation of $\mathbf{P}_{\mathbf{2}} \mathbf{W}_{\mathbf{1 8}}$ clusters into the pores of the NU-1000 host. High-resolution transmission electron microscopy (HR-TEM) images further display the successful incorporation of $\mathbf{P}_{\mathbf{2}} \mathbf{W}_{\mathbf{1 8}}$ clusters (deep dark dots shown in Fig. 1c) into the NU-1000 host (Fig. 1b).

Table 1 ICP-AES results of W/P, W/Zr, and $\mathrm{P}_{2} \mathrm{~W}_{18} /\left\{\mathrm{Zr}_{6}\right\}$ in the $\mathrm{P}_{2} \mathrm{~W}_{18} @ \mathrm{aNU}-1000$ composite

\begin{tabular}{|c|c|c|c|}
\hline Compounds & $\mathrm{W} / \mathrm{P}$ & $\mathrm{W} / \mathrm{Zr}$ & $\mathbf{P}_{2} \mathbf{W}_{\mathbf{1 8}} /\left\{\mathrm{Zr}_{6}\right\}$ \\
\hline $\begin{array}{l}0.25-\mathbf{P}_{2} \mathbf{W}_{18} @ N U- \\
1000\end{array}$ & 8.20 & 0.76 & 0.25 \\
\hline $\begin{array}{l}0.29-\mathbf{P}_{2} \mathbf{W}_{18} @ N U- \\
1000\end{array}$ & 8.15 & 0.88 & 0.29 \\
\hline $\begin{array}{l}0.32-\mathbf{P}_{2} \mathbf{W}_{18} @ N U- \\
1000\end{array}$ & 8.09 & 0.95 & 0.32 \\
\hline $\begin{array}{l}0.34-\mathbf{P}_{2} \mathbf{W}_{18} @ N U- \\
1000\end{array}$ & 8.10 & 1.03 & 0.34 \\
\hline
\end{tabular}



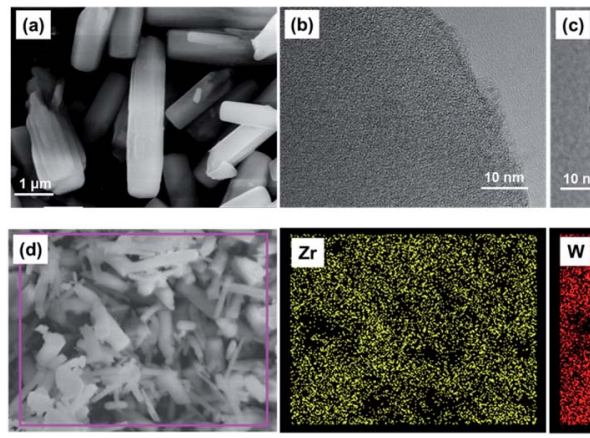

Fig. 1 Characterization of $\mathrm{NU}-1000$ and the $0.34-\mathrm{P}_{2} \mathrm{~W}_{18} @ \mathrm{NU}-1000$ composite. (a) SEM image of $0.34-\mathrm{P}_{2} \mathrm{~W}_{18} @ \mathrm{QNU}-1000$. HR-TEM images of (b) NU-1000 and (c) 0.34- $\mathrm{P}_{2} \mathrm{~W}_{18}(\mathrm{aNU}-1000$. (d) The corresponding elemental mapping images of $0.34-\mathrm{P}_{2} \mathrm{~W}_{18} @ \mathrm{NU}-1000$.

In addition, Energy Dispersive X-ray Spectroscopy (EDS) (Fig. S3 $\dagger$ ) and elements mapping images of 0.34- $\mathbf{P}_{\mathbf{2}} \mathbf{W}_{\mathbf{1 8}} @ N U$ 1000 (Fig. 1d) confirm the presence and uniform distribution of $\mathrm{Zr}$ and $\mathrm{W}$ elements of $\mathbf{P}_{\mathbf{2}} \mathbf{W}_{\mathbf{1 8}}$ in the NU-1000 host, further providing strong and straight evidence for the successful immobilization of $\mathbf{P}_{\mathbf{2}} \mathbf{W}_{\mathbf{1 8}}$.

Low temperature $\mathrm{N}_{2}$ adsorption-desorption tests for NU1000 and 0.34- $\mathbf{P}_{2} \mathbf{W}_{18} @ N U-1000$ were carried out to further reveal the incorporation and distribution of $\mathbf{P}_{\mathbf{2}} \mathbf{W}_{\mathbf{1 8}}$ in the NU1000 host (Fig. 2a). The calculated BET surface areas decreased from $2065.7 \mathrm{~m}^{2} \mathrm{~g}^{-1}$ for pristine NU-1000 to $670.6 \mathrm{~m}^{2}$ $\mathrm{g}^{-1}$ for the 0.34- $\mathbf{P}_{2} \mathbf{W}_{18} @ N U-1000$ composite, revealing the successful incorporation of $\mathbf{P}_{\mathbf{2}} \mathbf{W}_{\mathbf{1 8}}$ into the pores of NU-1000. The type IV hysteresis loop disappeared in the $\mathrm{N}_{2}$ adsorptiondesorption isotherm of NU-1000 after immobilization of $\mathbf{P}_{\mathbf{2}} \mathbf{W}_{\mathbf{1 8}}$, demonstrating that the typical mesopores have been blocked thereby leading to a decreased adsorbed volume. Meanwhile, the overlapped adsorption and desorption curves imply the dominating role of micropores in the porous structure of 0.34$\mathbf{P}_{\mathbf{2}} \mathbf{W}_{18} @ \mathrm{NU}$-1000. The pore distribution curves in Fig. 2b also confirm the above-mentioned changes in the texture properties of NU-1000 after the immobilization of $\mathbf{P}_{\mathbf{2}} \mathbf{W}_{\mathbf{1 8}}$. Given the molecular size of $\mathbf{P}_{\mathbf{2}} \mathbf{W}_{\mathbf{1 8}}$ (Scheme 1), the $\mathbf{P}_{\mathbf{2}} \mathbf{W}_{\mathbf{1 8}}$ clusters could also be encapsulated into the micropores of NU-1000 as confirmed by the pore distribution curve shown in Fig. 2 b.

Fourier transform infrared (FT-IR) spectra were employed to determine the physicochemical interactions between the $\mathbf{P}_{\mathbf{2}} \mathbf{W}_{\mathbf{1 8}}$
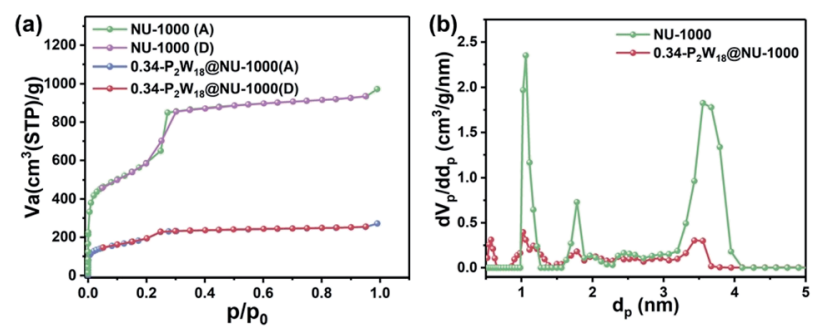

Fig. 2 (a) The low temperature $\mathrm{N}_{2}$ adsorption-desorption isotherms and $(b)$ the pore distribution curves of NU-1000 and 0.34- $\mathrm{P}_{2} \mathrm{~W}_{18} @ \mathrm{NU}-$ 1000. Note: A and D represent adsorption and desorption, respectively.
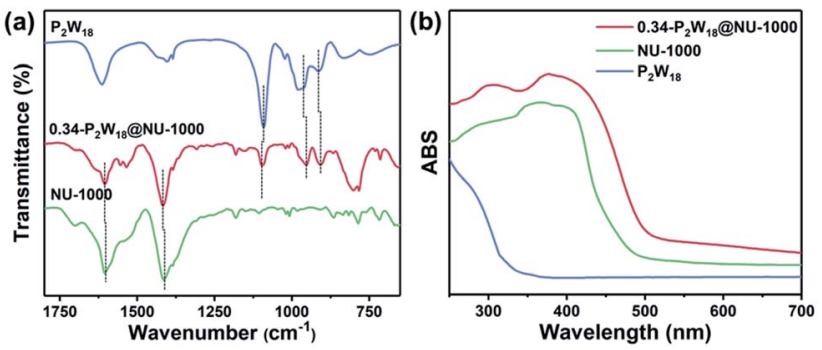

Fig. 3 (a) FT-IR spectra and (b) UV-Vis diffuse reflectance spectra of $\mathrm{P}_{2} \mathrm{~W}_{18}, \mathrm{NU}-1000$ and $0.34-\mathrm{P}_{2} \mathrm{~W}_{18} @ \mathrm{aNU}-1000$.

guest and NU-1000 host. The characteristic IR bands from 650 to $1200 \mathrm{~cm}^{-1}$ are assigned to the $\mathrm{Zr}-\mathrm{O}$ vibrational modes and carboxylate stretches in NU-1000, while the peaks from 700 to $1100 \mathrm{~cm}^{-1}$ are attributed to the typical $\mathrm{W}-\mathrm{O}$ and $\mathrm{P}-\mathrm{O}$ vibrational bands of $\mathbf{P}_{2} \mathbf{W}_{18}$ POM. The presence of $\mathrm{W}-\mathrm{O}$ and $\mathrm{P}-\mathrm{O}$ vibrational bands clearly confirms the successful incorporation of $\mathbf{P}_{\mathbf{2}} \mathbf{W}_{\mathbf{1 8}}$ into the $\mathbf{P}_{\mathbf{2}} \mathbf{W}_{\mathbf{1 8}} @ N U-1000$ composite (Fig. 3a). Additionally, their IR absorption characteristic peaks have a slight red shift upon $\mathbf{P}_{2} \mathbf{W}_{18}$ immobilization. For example, the characteristic peaks of $\mathrm{P}-\mathrm{O}$ and $\mathrm{W}-\mathrm{O}$ bands shifted from $1091.20 \mathrm{~cm}^{-1}$ and $912.93 \mathrm{~cm}^{-1}$ for bare $\mathbf{P}_{\mathbf{2}} \mathbf{W}_{\mathbf{1 8}}$ to $1095.48 \mathrm{~cm}^{-1}$ and $907.04 \mathrm{~cm}^{-1}$ for the $\mathbf{P}_{\mathbf{2}} \mathbf{W}_{\mathbf{1 8}} @ \mathrm{NU}-1000$ composite, respectively. The slight shifts of IR characterization peaks would be attributed to the electronic interaction between the closely contacted $\mathbf{P}_{\mathbf{2}} \mathbf{W}_{\mathbf{1 8}}$ guest and NU-1000 host instead of the simple mechanical mixture. UV-Vis diffuse reflectance spectra revealed the changes in optical properties upon immobilization (Fig. 3b). It is observed that the $\mathbf{P}_{\mathbf{2}} \mathbf{W}_{\mathbf{1 8}} @ N U-1000$ composite displays both enhanced absorption ability and an extended absorption edge to a longer wavelength in the visible light region, further demonstrating the electronic interaction between $\mathbf{P}_{\mathbf{2}} \mathbf{W}_{\mathbf{1 8}}$ and NU-1000.

The detailed photocatalytic hydrogen production measurements are described in the Experimental section. We first investigated the effects of $\mathbf{P}_{\mathbf{2}} \mathbf{W}_{\mathbf{1 8}}$ loading quantity, $\mathrm{pH}$ value, and the addition amount of $\mathrm{Pt}$ to achieve optimal photocatalytic conditions. With the increasing feedstock of $\mathbf{P}_{\mathbf{2}} \mathbf{W}_{\mathbf{1 8}}$ during the synthesis, the immobilized content of $\mathbf{P}_{\mathbf{2}} \mathbf{W}_{\mathbf{1 8}}$ into NU-1000 gradually increases (Table 1). Among our tested synthetic samples, the 0.34- $\mathbf{P}_{2} \mathbf{W}_{\mathbf{1 8}} @ N U-1000$ composite achieves the highest photocatalytic activity, indicating that the presence of $\mathbf{P}_{\mathbf{2}} \mathbf{W}_{\mathbf{1 8}}$ could facilitate the separation of photogenerated charge carriers in the NU-1000 host and the subsequent electron transfer for hydrogen production (Fig. 4a). The hydrogen production yield exhibits a typical volcano behavior versus $\mathrm{pH}$ values (Fig. S4†). The optimal $\mathrm{pH}$ value was achieved at 5.5, which could be ascribed to the following reasons: (1) a very low $\mathrm{pH}$ value might cause the destruction of the $\mathbf{P}_{\mathbf{2}} \mathbf{W}_{\mathbf{1 8}} @ \mathrm{NU}-1000$ structure, (2) the sacrificial electron donor AA shows the best performance at $\mathrm{pH} 5.5$, and (3) an overly high $\mathrm{pH}$ value is thermodynamically unfavorable for hydrogen generation. Similarly, the moles of hydrogen production also display the volcano shape versus the added Pt in terms of weight percentage (Fig. 4b). The photocatalytic system of 0.34- $\mathbf{P}_{\mathbf{2}} \mathbf{W}_{\mathbf{1 8}} @ \mathrm{NU}-1000$ 

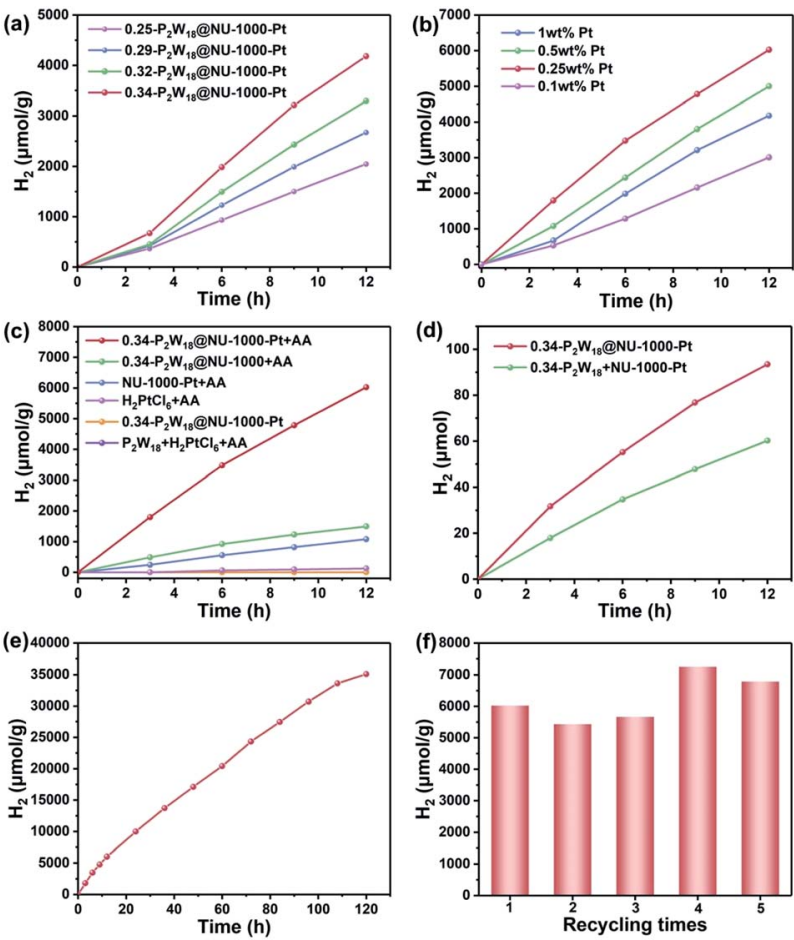

Fig. 4 (a) Photocatalytic hydrogen evolution of $\mathrm{P}_{2} \mathrm{~W}_{18} @ \mathrm{NU}-1000$ with different molar ratios of $\mathrm{P}_{2} \mathrm{~W}_{18} / \mathrm{NU}-1000$. Conditions: $10 \mathrm{mg}$ catalysts, $\mathrm{pH}=4.5,1$ wt\% Pt. (b) Photocatalytic hydrogen evolution tests using

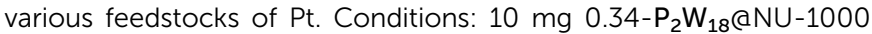
photocatalyst, $\mathrm{pH}=5.5$. (c) Comparison profiles of photocatalytic hydrogen production with different catalysts and reaction conditions (the specific content of $\mathrm{Pt}$ contained in the reaction systems is 0.25 wt\%). The common conditions: $\mathrm{pH}=5.5,300 \mathrm{~W}$ Xe-lamp. (d) The comparison profiles of the photocatalytic hydrogen production for 0.34- $\mathrm{P}_{2} \mathrm{~W}_{18} \mathrm{aNU}-1000$ and $\mathrm{P}_{2} \mathrm{~W}_{18}+\mathrm{NU}-1000$. Conditions: $\mathrm{pH}=5.5$, $0.25 \mathrm{wt} \% \mathrm{Pt}$. The contents of $\mathrm{P}_{2} \mathrm{~W}_{18}$ and $\mathrm{NU}-1000$ are equal to that of 0.34- $\mathrm{P}_{2} \mathrm{~W}_{18} @ \mathrm{NNU}-1000$. (e) Long-term photocatalytic hydrogen production test using the $0.34-\mathrm{P}_{2} \mathrm{~W}_{18} @ \mathrm{NU}-1000$ composite with $0.25 \mathrm{wt} \% \mathrm{Pt}$ at $\mathrm{pH}=5.5$. (f) The photocatalytic recycle tests of the 0.34- $\mathrm{P}_{2} \mathrm{~W}_{18} @ \mathrm{NU}-1000$ photocatalyst. Conditions: $10 \mathrm{mg} 0.34-$ $\mathrm{P}_{2} \mathrm{~W}_{18} @ \mathrm{aNU}-1000$ photocatalyst, $0.25 \mathrm{wt} \% \mathrm{Pt}, 300 \mathrm{~W}$ Xe-lamp, and $20 \mathrm{~mL}$ of $1 \mathrm{M} \mathrm{AA}$ aqueous at $\mathrm{pH}=5.5$.

with 0.25 wt $\%$ Pt dosage exhibits the best performance for hydrogen production, achieving $6023 \mu \mathrm{mol} \mathrm{g}^{-1}$ hydrogen production with an apparent quantum yield of $1.69 \%$ (see calculation details in the ESI $\dagger$ ) after $12 \mathrm{~h}$ illumination under optimized conditions. When the $\mathrm{Pt}$ dosage is lower than 0.25 wt $\%$, the synergistic catalysis between $\mathbf{P}_{\mathbf{2}} \mathbf{W}_{\mathbf{1 8}}$ and Pt NPs is relatively weak; in contrast, addition of too high amount of $\mathrm{Pt}$ could not only block the channels of the NU-1000 host but also cause light scattering that are detrimental to efficient hydrogen evolution. Therefore, subsequent studies were performed under optimized conditions $(\mathrm{pH}=5.5,0.25 \mathrm{wt} \% \mathrm{Pt}, 10 \mathrm{mg}$ of $0.34-$ $\mathbf{P}_{\mathbf{2}} \mathbf{W}_{18} @$ @U-1000 composite) unless otherwise noted.

Based on the above experimental analyses, we can speculate that such superior photocatalytic activity of the three-in-one $\mathbf{P}_{\mathbf{2}} \mathbf{W}_{18} @ N U-1000-\mathrm{Pt}$ hybrid composite is attributed to the enhanced photo-response, efficient electron transfer between the NU-1000 host and $\mathbf{P}_{\mathbf{2}} \mathbf{W}_{\mathbf{1 8}}$ guest, and the suitable amount of in situ generated Pt NP co-catalyst. To further prove this hypothesis, we carried out various control experiments under otherwise identical conditions (Fig. 4c). Clearly, NU-1000, the sacrificial donor, $\mathbf{P}_{\mathbf{2}} \mathbf{W}_{\mathbf{1 8}}$, and the in situ generated Pt NPs are indispensable for efficient photocatalytic hydrogen production. The control experiment in the absence of NU-1000 (purple curve, Fig. 4c) reveals that the absorption of photons by the photo-responsive NU-1000 is a prerequisite for the photocatalytic reaction. The negligible hydrogen production without AA (yellow curve, Fig. 4c) indicates the necessity of a sacrificial agent (AA) in this photocatalytic system. Moreover, the remarkably decreased hydrogen yields for NU-1000-Pt + AA (blue curve, Fig. 4c) and 0.34- $\mathbf{P}_{\mathbf{2}} \mathbf{W}_{\mathbf{1 8}} @ \mathrm{NU}-1000+\mathrm{AA}$ (green curve, Fig. 4c) systems indicate that the $\mathbf{P}_{\mathbf{2}} \mathbf{W}_{\mathbf{1 8}}$ clusters and the in situ generated Pt NPs derived from $\mathrm{H}_{2} \mathrm{PtCl}_{6}$ reduction are also crucial for efficient photocatalysis. As electron-storage sponge, the outstanding reversible multi-electron-storing ability of $\mathbf{P}_{\mathbf{2}} \mathbf{W}_{\mathbf{1 8}}$ clusters could facilitate effective separation of photogenerated charge carriers, thereby leading to subsequently efficient hydrogen production. ${ }^{38}$ The Pt NPs could be in situ generated in the NU-1000-Pt + AA catalytic system, which was confirmed by the high-resolution TEM image (Fig. S5†) and XPS signals of Pt $4 \mathrm{f}$ after photocatalysis (Fig. S6†). The Pt $4 \mathrm{f}$ XPS signal could be deconvoluted into two species, corresponding to metallic Pt NPs and $\mathrm{Pt}^{2+}$ states, respectively, originating from the photoreduction of $\mathrm{H}_{2} \mathrm{PtCl}_{6}$ during photocatalysis. The in situ generated Pt NPs (Step (II) in Scheme 1) could also be evidenced by the EDS analysis and elemental mapping images of 0.34$\mathbf{P}_{2} \mathbf{W}_{18} @ N U-1000$-Pt after photocatalysis (Fig. S7 and S8 $\dagger$ ). Additionally, the low temperature $\mathrm{N}_{2}$ adsorption-desorption isotherms and the changes in pore distribution further proved the in situ formation of Pt NPs during the photocatalytic process (Fig. S9 and S10 $\dagger$ ). To further evaluate the importance of the in situ formed Pt NPs, we compared the photocatalytic activities using commercially available platinum-carbon (Pt/C) and synthesized cubic Pt NPs under otherwise identical conditions (Fig. S11 $\dagger$ ). The hydrogen production dramatically decreases using these externally added catalysts, which strongly identifies the importance of close contact between in situ generated Pt NPs, $\mathbf{P}_{\mathbf{2}} \mathbf{W}_{\mathbf{1 8}}$ clusters, and the NU-1000 host in the three-in-one $\mathbf{P}_{\mathbf{2}} \mathbf{W}_{18} @$ NU-1000-Pt hybrid composite. Moreover, the catalytic system using the mechanical mixture $\left(\mathbf{P}_{\mathbf{2}} \mathbf{W}_{\mathbf{1 8}}+\mathrm{NU}-1000-\mathrm{Pt}\right)$ shows obviously declined activity, further confirming that the hydrogen production largely depends on the strong electronic interaction between the $\mathbf{P}_{\mathbf{2}} \mathbf{W}_{\mathbf{1 8}}$ guest and NU-1000 host (Fig. $4 \mathrm{~d}$ ).

To evaluate the stability of the three-in-one hybrid composite 0.34-P $\mathbf{P}_{2} \mathbf{W}_{18} @ N U-1000-\mathrm{Pt}$, we have performed a long-term photocatalytic hydrogen evolution test under the optimal experimental conditions (Fig. 4e). Upon irradiation with a full spectrum $300 \mathrm{~W}$ Xe-lamp for $120 \mathrm{~h}$, such a three-in-one hybrid photocatalyst maintains a satisfactory photocatalytic activity with hydrogen production as high as $35100 \mu \mathrm{mol} \mathrm{g}^{-1}$, corresponding to a TON of 5484 versus moles of $\mathrm{Pt}$, which belongs to the top performance compared to similar catalytic systems in the literature (Table S1 $\dagger$ ). Additionally, the morphology and crystalline structure of the 0.34- $\mathbf{P}_{2} \mathbf{W}_{\mathbf{1 8}} @ N U-1000$ composite remain largely unchanged after photocatalysis as confirmed by 
SEM images (Fig. S12 $\dagger$ ), PXRD patterns (Fig. S13 $\dagger$ ) and FT-IR spectra (Fig. S14 $\dagger$ ). We also conducted the recycling stability test of the three-in-one hybrid photocatalyst (Fig. 4f), which could be easily recycled at least 5 times without declining catalytic activity even though there are around $2.1 \% \mathbf{P}_{2} \mathbf{W}_{18}$ clusters and $0.5 \% \mathrm{Pt}$ species leaching out into the reaction solution after first-cycle photocatalysis as confirmed by ICP-AES measurements. The slightly increased hydrogen production after 3 cycles could have resulted from the better dispersion of the photocatalyst under a prolonged reaction time, leading to better utilization of absorbed photons. Given the strong absorption of $\mathbf{P}_{\mathbf{2}} \mathbf{W}_{\mathbf{1 8}} @ \mathrm{NU}-1000$ composites in the UV light region, it is rational that the photocatalytic activity is better under full-optical irradiation with a $300 \mathrm{~W}$ Xe-lamp than under only visible light irradiation (Fig. S15†). But the extended visible light absorption of $\mathbf{P}_{\mathbf{2}} \mathbf{W}_{\mathbf{1 8}} @ \mathrm{NU}-1000$ provides decent photocatalytic activity under visible light $(>420 \mathrm{~nm})$ compared to that of pristine NU-1000 and $\mathbf{P}_{2} \mathbf{W}_{\mathbf{1 8}}$. Such enhanced photoresponsive ability enables the potential of $\mathbf{P}_{\mathbf{2}} \mathbf{W}_{\mathbf{1 8}} @ \mathrm{NU}-1000$ to utilize natural sunlight to drive hydrogen evolution. As shown in Fig. S16, $\uparrow$ the yield of hydrogen production increases with irradiation time even in a cloudy weather, but it varies with UV light intensity during the daytime. These results demonstrate the potential practical value of our three-in-one $\mathbf{P}_{2} \mathbf{W}_{\mathbf{1 8}} @ \mathrm{NU}$ 1000-Pt photocatalyst in the utilization of real natural solar energy.

To deeply elucidate the photocatalytic mechanism, we have conducted a series of characterization studies for NU-1000 and $\mathbf{P}_{2} \mathbf{W}_{18} @ N U-1000$ composites, respectively. Fig. 5a shows the steady-state photoluminescence (PL) spectra of pristine NU1000 and the 0.34- $\mathbf{P}_{2} \mathbf{W}_{18} @ N U-1000$ composite upon excitation with $365 \mathrm{~nm}$ laser light. An obvious strong PL signal is found for pristine NU-1000 with the maximum emission at $\sim 505 \mathrm{~nm}$ (red curve, Fig. 5a), while it is effectively quenched after immobilization of $\mathbf{P}_{\mathbf{2}} \mathbf{W}_{\mathbf{1 8}}$ in the 0.34- $\mathbf{P}_{\mathbf{2}} \mathbf{W}_{\mathbf{1 8}} @ \mathrm{NU}-1000$ composite (green curve, Fig. 5a), implying the efficient transfer of photoinduced electrons from the photoexcited NU-1000 host to the $\mathbf{P}_{\mathbf{2}} \mathbf{W}_{\mathbf{1 8}}$ cluster. The charge carrier transfer dynamics was further determined by time-resolved PL measurements on pristine NU1000 and 0.34- $\mathbf{P}_{\mathbf{2}} \mathbf{W}_{\mathbf{1 8}} @ \mathrm{NU}-1000$ (Fig. 5b). The lifetimes were estimated by fitting the PL decay kinetics data with a threeexponential decay function, and are summarized in Table S2. $\dagger$ Clearly, the immobilization of $\mathbf{P}_{\mathbf{2}} \mathbf{W}_{\mathbf{1 8}}$ clusters into the NU-1000
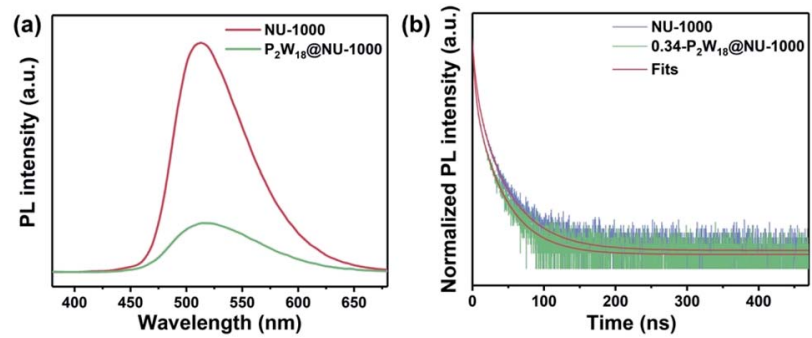

Fig. 5 (a) Photoluminescence spectra and (b) normalized timeresolved PL decay kinetics ( $\lambda_{\mathrm{em}}=505 \mathrm{~nm}$ ) of $\mathrm{NU}-1000$ and 0.34 $\mathrm{P}_{2} \mathrm{~W}_{18} @ \mathrm{NU}-1000$ solid samples upon excitation at $365 \mathrm{~nm}$.
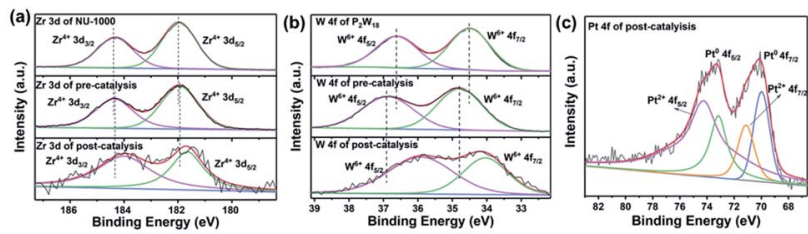

(d)

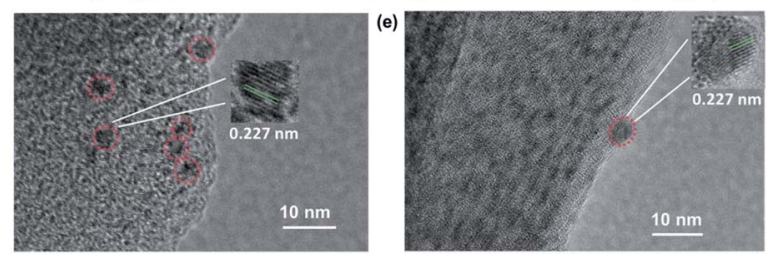

Fig. 6 High resolution XPS spectra of (a) Zr 3d and (b) W $4 \mathrm{f}$ signals in $\mathrm{NU}-1000$ and $0.34-\mathrm{P}_{2} \mathrm{~W}_{18} @ \mathrm{NU}-1000$ before and after photocatalysis. (c) High resolution XPS spectra of Pt 4 f signals in $0.34-\mathrm{P}_{2} \mathrm{~W}_{18} \mathrm{QNU}-$ 1000 after photocatalysis. (d and e) HR-TEM images of 0.34$\mathrm{P}_{2} \mathrm{~W}_{18}$ @NU-1000 after photocatalysis. The in situ formed Pt NPs located into the pores or on the surface of NU-1000 are marked with red circles.

host obviously decreases the PL decay lifetime, further proving the effective transfer of photogenerated charge carriers from the excited NU-1000 host to the $\mathbf{P}_{\mathbf{2}} \mathbf{W}_{\mathbf{1 8}}$ guest.

In addition, the XPS technique was further employed to determine the changes in the chemical states of elements for the 0.34- $\mathbf{P}_{2} \mathbf{W}_{18} @ N U-1000$ composite before and after photocatalysis. The binding energy of $\mathrm{Zr}$ in NU-1000 shifts negatively a bit while the binding energy of $\mathrm{W}$ shifts positively after incorporation into NU-1000, which could be attributed to the slight electron density movement from $\mathbf{P}_{\mathbf{2}} \mathbf{W}_{\mathbf{1 8}}$ to the $\left\{\mathrm{Zr}_{6}\right\}$ nodes of NU-1000 (Fig. 6a and b). Such experimental evidence confirms the strong electronic interaction between the NU-1000 host and the $\mathbf{P}_{\mathbf{2}} \mathbf{W}_{\mathbf{1 8}}$ cluster as evidenced by the results of steadystate and time-resolved photoluminescence spectra (Fig. 5). After photocatalysis, both the binding energies of $\mathrm{Zr}$ and $\mathrm{W}$ shift negatively, indicating the photoreduction of $\mathrm{Zr}$ and $\mathrm{W}$ centers under a strong reducing environment during photocatalysis (Fig. 6a and b). Moreover, the significant XPS signals of reduced Pt species $\left(\mathrm{Pt}^{0}\right.$ and $\left.\mathrm{Pt}^{2+}\right)$ also strongly confirmed that the Pt NPs are generated in situ from the reduction of $\mathrm{H}_{2} \mathrm{PtCl}_{6}$ during photocatalysis (Fig. 6c). The ICP-AES technique was employed to determine the amount of homogeneous $\left[\mathrm{PtCl}_{6}\right]^{2-}$ remaining in the supernatant after one-cycle photocatalysis $(12 \mathrm{~h})$, and the results revealed that around $0.5 \% \mathrm{Pt}$ species still remained in the post-reaction solution. The in situ formed Pt NPs are located both on the surface and in the pores of NU-1000 that was confirmed by HR-TEM images of the composite after photocatalysis with the characteristic crystal lattice spacing $(0.227$ $\mathrm{nm}$ ) of Pt NPs (Fig. 6d and e). These results also illustrated that in situ generated Pt NPs could work as the active hydrogenevolving sites during photocatalytic reactions.

Based on the above observations and analyses in detail, we have drawn a schematic energy level diagram ${ }^{66,67}$ for photocatalytic hydrogen production using the three-in-one $\mathbf{P}_{\mathbf{2}} \mathbf{W}_{\mathbf{1 8}} @$ NU-1000-Pt photocatalyst (Fig. 7). Herein, the LUMO positions of the $\mathbf{P}_{\mathbf{2}} \mathbf{W}_{\mathbf{1 8}}$ cluster were measured by using cyclic 


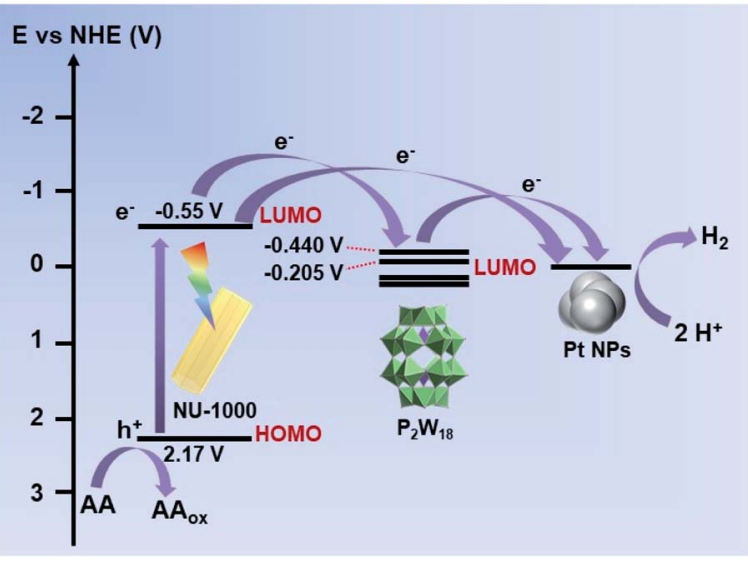

Fig. 7 Schematic energy level diagram of photocatalytic hydrogen production using the three-in-one $\mathrm{P}_{2} \mathrm{~W}_{18} \mathrm{aNU}-1000-\mathrm{Pt}$ photocatalyst.

voltammograms (Fig. S17 $\dagger$ ), and the potentials at $-0.205 \mathrm{~V}$ and $-0.440 \mathrm{~V} v s$. NHE correspond to the LUMO positions of threeand four-electrons reduced $\mathbf{P}_{\mathbf{2}} \mathbf{W}_{\mathbf{1 8}}$, respectively, which is supported by the experimental observation of heteropoly blue (reduced $\mathbf{P}_{2} \mathbf{W}_{\mathbf{1 8}}$ clusters) as shown in Fig. S16b. $\dagger$ According to the schematic energy level diagram, a systematic photocatalytic mechanism could be speculated as follows. Upon light irradiation, the photo-responsive NU-1000 MOF host can efficiently harvest incident photons to generate photoexcited states. Subsequently, the photoexcited NU-1000 host could be subsequently quenched by transferring photogenerated electrons to the immobilized $\mathbf{P}_{\mathbf{2}} \mathbf{W}_{\mathbf{1 8}}$ POM cluster to generate the reduced POM $\left(\mathrm{POM}_{\mathrm{red}}\right)$ as indicated by the blue solution color in Fig. S16b. $\dagger$ Meanwhile, the photogenerated electrons can also reduce $\mathrm{H}_{2} \mathrm{PtCl}_{6}$ in solution to in situ generate Pt NPs. At the same time, the oxidized states of the NU-1000 MOF could be reduced back to the initial state by the sacrificial reagent (AA). Herein, either $\mathbf{P}_{2} \mathbf{W}_{\mathbf{1 8}}$ clusters or Pt NPs alone could catalyze hydrogen production (green and blue curves, Fig. 4c), but the hydrogen production efficiency can be greatly enhanced while the NU-1000 MOF, $\mathbf{P}_{\mathbf{2}} \mathbf{W}_{\mathbf{1 8}}$ cluster, and Pt NPs work synergistically together as a three-in-one $\mathbf{P}_{2} \mathbf{W}_{\mathbf{1 8}} @ \mathrm{NU}-1000-\mathrm{Pt}$ photocatalyst (red curve, Fig. 4c). Overall, the outstanding photocatalytic activity of such a three-in-one $\mathbf{P}_{\mathbf{2}} \mathbf{W}_{\mathbf{1 8}} @ \mathrm{NU}-1000-$ Pt hybrid photocatalyst could be ascribed to the synergistic effect of the good photo-absorbing properties of NU-1000, the reversible multi-electron-transfer ability of the $\mathbf{P}_{\mathbf{2}} \mathbf{W}_{\mathbf{1 8}}$ catalyst, and the superior catalytic activity of closely contacted Pt NPs generated in situ under turnover conditions.

\section{Conclusions}

In summary, we have prepared a three-in-one hybrid material $\left(\mathbf{P}_{\mathbf{2}} \mathbf{W}_{18} @ \mathrm{NU}-1000-\mathrm{Pt}\right)$ through the strong host-guest interaction using a facile impregnation and subsequent in situ photoreduction approach. A series of physicochemical techniques confirmed the successful immobilization of $\mathbf{P}_{\mathbf{2}} \mathbf{W}_{\mathbf{1 8}}$ clusters and the strong electronic interaction between the $\mathbf{P}_{\mathbf{2}} \mathbf{W}_{\mathbf{1 8}}$ guest and NU-1000 host.
Under minimally optimized photocatalytic conditions, the three-inone 0.34- $\mathbf{P}_{2} \mathbf{W}_{18} @ N U-1000-P t$ hybrid composite exhibits efficient photocatalytic activity with the hydrogen production of 35100 $\mu \mathrm{mol} \mathrm{g}^{-1}$ after 5 days of reaction. The post-catalysis hybrid composite could be easily recycled at least 5 times without declining catalytic activity. Based on the solid analyses of various spectroscopic and experimental results, we concluded that the outstanding and robust photocatalytic activity of this three-in-one $\mathbf{P}_{2} \mathbf{W}_{18} @ N U-1000-P t$ hybrid composite could be ascribed to the synergistic effect of the good photo-absorbing properties of NU1000 , the reversible multi-electron-transfer ability of the $\mathbf{P}_{\mathbf{2}} \mathbf{W}_{\mathbf{1 8}}$ catalyst, and the superior catalytic activity of closely contacted Pt NPs generated in situ under turnover conditions. More importantly, the present water-compatible photocatalytic system can also effectively catalyze hydrogen production under natural sunlight irradiation even in a cloudy weather, demonstrating the potential practical value for natural solar energy utilization. Considering the feasibility of the synthetic strategy, robustness and effectiveness of the resulting three-in-one hybrid materials, many interesting functional materials could be constructed based on POM@MOF architectures by following a similar idea in our work.

\section{Experimental section}

\section{Materials and methods}

All reagents and solvents are purchased from commercial sources without further purification. EDX spectra and SEM images are obtained on a ZEISS supra55. HRTEM images are obtained on a JEOL JEM-2100. PXRD patterns are collected on a MiniFlex 600 diffractometer with a $\mathrm{Cu}-\mathrm{K} \alpha$ X-ray radiation source. Low temperature $\mathrm{N}_{2}$ adsorption-desorption isotherms are measured at $77 \mathrm{~K}$ on a Quantachrome Instruments ASiQMVH002-5 after pre-treatment by activating the samples under vacuum at $120^{\circ} \mathrm{C}$ for $12 \mathrm{~h}$. UV-Vis spectra are recorded on a Techcomp UV 2600 spectrophotometer. FT-IR spectra are recorded on a Bruker Tensor II with $\mathrm{KBr}$ pellets. Fluorescence spectra are recorded with an Edinburgh Instruments Spectrofluorometer FS5. ICP-AES data are obtained with a Spectro Arcos EOP (Axial View Inductively Coupled Plasma Spectrometer). XPS data are collected by using a PHI 5000 VersaProbe III Scanning XPS Microprobe. Gas chromatograph analysis is conducted with a Techcomp GC7900 gas chromatograph equipped with a thermal conductivity detector. Cyclic voltammetry was recorded on a CHI660E Electrochemical potentiostat with a threeelectrode setup using a glassy carbon working electrode, saturated calomel electrode (SCE) reference electrode, and Pt wire auxiliary electrode. The working electrode was polished with 0.3 $\mu \mathrm{m}$ alumina, sonicated with deionized water, rinsed with EtOH and then with deionized water before use. The measured potential was calibrated by converting to the normal hydrogen electrode (NHE) scale.

\section{Synthetic procedures}

Synthesis of $\mathrm{K}_{6}\left[\boldsymbol{\alpha}-\mathrm{P}_{\mathbf{2}} \mathbf{W}_{\mathbf{1 8}} \mathrm{O}_{\mathbf{6 2}}\right] \cdot \mathbf{1 4 H}_{\mathbf{2}} \mathrm{O}$. The typical synthesis of $\mathbf{P}_{2} \mathbf{W}_{18}$ is as follows according to the reported literature.$^{68} 300 \mathrm{~g}$ of $\mathrm{Na}_{2} \mathrm{WO}_{4} \cdot 2 \mathrm{H}_{2} \mathrm{O}$ is dissolved in $350 \mathrm{~mL}$ deionized water. $4 \mathrm{M}$ 
$\mathrm{HCl}(250 \mathrm{~mL}, 1.00 \mathrm{~mol})$ is added dropwise to the above clear solution followed by adding $4 \mathrm{M} \mathrm{H}_{3} \mathrm{PO}_{4}(250 \mathrm{~mL}, 1.00 \mathrm{~mol})$. The resulting pale-yellow solution is subsequently refluxed for $24 \mathrm{~h}$. After cooling down to room temperature, $150 \mathrm{~g} \mathrm{KCl}$ is added into the resultant bright yellow solution under vigorous stirring to obtain the yellow precipitate. This crude product is then dissolved in $650 \mathrm{~mL}$ water which pre-contained 2 drops of $\mathrm{Br}_{2}$. The yellow solution is filtered to remove insoluble impurities. Finally, the solution is heated at $80{ }^{\circ} \mathrm{C}$ for $72 \mathrm{~h}$ and then proceeded to recrystallize in a $4{ }^{\circ} \mathrm{C}$ refrigerator.

Preparation of 1,3,6,8-tetrakis(4-(methoxycarbonyl)phenyl) pyrene (TBAPy). The synthesis method of TBAPy is described as follows based on the previous literature.$^{69,70} \mathrm{~A}$ mixture of (4(methoxycarbonyl)phenyl)boronic acid $(2.080 \mathrm{~g}, 11.6 \mathrm{mmol})$, 1,3,6,8-tetrabromopyrene $(1.000 \mathrm{~g}, 1.94 \mathrm{mmol})$, tetrakis(triphenylphosphine)palladium $(0.06 \mathrm{~g}, 0.026 \mathrm{mmol})$, and potassium tribasic phosphate $(2.200 \mathrm{~g}, 10.6 \mathrm{mmol})$ is dissolved into dry dioxane $(40 \mathrm{~mL})$ and then transferred to a $100 \mathrm{~mL}$ threenecked flask. The following detailed and modified method is described in previous work. ${ }^{37}$

Preparation of 1,3,6,8-tetrakis( $p$-benzoic acid)pyrene ( $\mathbf{H}_{4}$ TBAPy). The method of preparing $\mathrm{H}_{4}$ TBAPy using TBAPy is based on the reported literature and our previous work. ${ }^{37,69,70}$

Synthesis of $\mathrm{Zr}_{6}\left(\mu_{3}-\mathrm{OH}\right)_{\mathbf{8}}(\mathrm{OH})_{\mathbf{8}}(\mathrm{TBAPy})_{2} \quad$ (NU-1000). The synthetic procedure is as follows based on the reported literature: $192 \mathrm{mg}$ of $\mathrm{ZrOCl} \cdot 8 \mathrm{H}_{2} \mathrm{O}(0.60 \mathrm{mmol})$ and $5400 \mathrm{mg}(44$ $\mathrm{mmol}$ ) of benzoic acid are mixed and dissolved in $16 \mathrm{~mL}$ of DMF. The clear solution is incubated in an oven at $100{ }^{\circ} \mathrm{C}$ for $1 \mathrm{~h}$. The following synthetic method of NU-1000 is based on the reported literature and our previous work. ${ }^{37,69}$

Preparation of cubic platinum nanoparticles. Pt nanoparticles are synthesized by a colloidal method by using sodium polyacrylate as a capping agent and $\mathrm{K}_{2} \mathrm{PtCl}_{4}$ as a metallic precursor. ${ }^{71} 4.25 \mathrm{mg}$ of $\mathrm{K}_{2} \mathrm{PtCl}_{4}$ is dissolved in $100 \mathrm{~mL}$ of polyacrylate aqueous $(0.2 \mathrm{mM})$. The resulting colloidal suspension is purged with Ar gas for 20 min and promptly bubbled with $\mathrm{H}_{2}$ gas for $5 \mathrm{~min}$ in a round-bottom flask. The sealed flask is placed at room temperature for a few days to reduce the Pt NPs.

Preparation of the $\mathbf{P}_{\mathbf{2}} \mathbf{W}_{\mathbf{1 8}} @ \mathrm{NU}-\mathbf{1 0 0 0}$ composite. The preparation route is illustrated in Step (I) of Scheme 1 and the detailed procedures are described as follows. A certain amount of $\mathbf{P}_{2} \mathbf{W}_{18}\left(55.8 \mathrm{mg}(0.0115 \mathrm{~mol})\right.$ for $0.25-\mathbf{P}_{\mathbf{2}} \mathbf{W}_{\mathbf{1 8}} @ \mathrm{NU}-1000$, $111.6 \mathrm{mg}(0.023 \mathrm{~mol})$ for $0.29-\mathbf{P}_{\mathbf{2}} \mathbf{W}_{\mathbf{1 8}} @ \mathrm{NU}-1000,223.3 \mathrm{mg}$ $(0.046 \mathrm{~mol})$ for 0.32-P $\mathbf{W}_{18} @ N U-1000,446.4 \mathrm{mg}(0.092 \mathrm{~mol})$ for 0.34-P $\left.\mathbf{P}_{\mathbf{2}} \mathbf{W}_{\mathbf{1 8}} @ \mathrm{NU}-1000\right)$ is completely dissolved in $10 \mathrm{~mL}$ deionized water, followed by the addition of NU-1000 $(50 \mathrm{mg}, 0.023$ $\mathrm{mol}$ ) and sonication for $20 \mathrm{~min}$. The resulting suspension liquid is stirred at room temperature for $72 \mathrm{~h}$ and followed by washing with water and acetone twice, respectively. Afterwards the sample is immersed in acetone for $12 \mathrm{~h}$ followed by centrifugation. Finally, the wet sample is dried at $80{ }^{\circ} \mathrm{C}$ under vacuum for $4 \mathrm{~h}$ to obtain the dark yellow powder denoted as $\mathbf{P}_{\mathbf{2}} \mathbf{W}_{\mathbf{1 8}} @ \mathrm{NU}$ 1000.

Hydrogen evolution test. Photocatalytic hydrogen evolution is conducted in a sealed glass reactor equipped with a quartz window and a magnetic stirrer. The typical photocatalytic hydrogen evolution test is described as follows. $20 \mathrm{~mL}$ of $1 \mathrm{M}$ ascorbic acid aqueous (AA) is first prepared. $10 \mathrm{mg}$ of the $\mathbf{P}_{2} \mathbf{W}_{18} @ N U-1000$ photocatalyst and $515 \mu \mathrm{L}$ of $1 \mathrm{mM} \mathrm{H}_{2} \mathrm{PtCl}_{6}$ aqueous are added into the aforesaid AA solution and homogeneously dispersed under magnetic stirring. The mixture was degassed with $\mathrm{Ar} / \mathrm{CH}_{4}$ (4/1) to remove air, where $\mathrm{CH}_{4}$ was used as an internal standard. A $300 \mathrm{~W}$ Xe-lamp (Beijing Perfectlight PLS-SXE300D) with a $420 \mathrm{~nm}$ filter is utilized as a light source. The reaction temperature is maintained at $25{ }^{\circ} \mathrm{C}$ through the air-cooling system. Under irradiation, $\mathbf{P}_{\mathbf{2}} \mathbf{W}_{\mathbf{1 8}} @ \mathrm{NU}$ 1000 absorbs ultraviolet light and part of the visible light $(<500$ $\mathrm{nm}$ ) and generates photo-induced electrons and holes. The holes are subsequently consumed by the sacrificial agent of AA, while the electrons are used to reduce $\mathbf{P}_{2} \mathbf{W}_{\mathbf{1 8}}$ and $\mathrm{H}_{2} \mathrm{PtCl}_{6}$ to in situ generate reduced $\mathbf{P}_{\mathbf{2}} \mathbf{W}_{\mathbf{1 8}}$ and Pt NPs, which subsequently reduced protons to $\mathrm{H}_{2}$ gas. The produced hydrogen is thus analyzed with a gas chromatograph (Techcomp GC7900) with an I Molecular sieve, 5 A packed column $(60 / 80$ mesh $2 \mathrm{~m}(\mathrm{~L}) \times$ $3 \mathrm{~mm}(\mathrm{OD}) \times 2 \mathrm{~mm}(\mathrm{ID}))$ and $\mathrm{Ar}$ as the carrier gas.

\section{Author contributions}

Le Jiao: methodology, data curation, formal analysis, investigation, writing - original draft preparation. Yuanyuan Dong: formal analysis, writing - reviewing and editing. Xing Xin: software. Ruijie Wang: data curation. Hongjin Lv: funding acquisition, project administration, conceptualization, supervision, validation, writing - reviewing and editing.

\section{Conflicts of interest}

There are no conflicts of interest to declare.

\section{Acknowledgements}

This work is financially supported by the National Natural Science Foundation of China (21871025 and 21831001), the Recruitment Program of Global Experts (Young Talents) and the BIT Excellent Young Scholars Research Fund.

\section{References}

1 L. Cui, J. Song, A. F. McGuire, S. Kang, X. Fang, J. Wang, C. Yin, X. Li, Y. Wang and B. Cui, ACS Nano, 2018, 12, 5551-5558.

2 Y. Kang, Y. Yang, L. C. Yin, X. Kang, G. Liu and H. M. Cheng, Adv. Mater., 2015, 27, 4572-4577.

3 A. Indra, A. Acharjya, P. W. Menezes, C. Merschjann, D. Hollmann, M. Schwarze, M. Aktas, A. Friedrich, S. Lochbrunner, A. Thomas and M. Driess, Angew. Chem., Int. Ed., 2017, 56, 1653-1657.

4 Q. Li, B. Guo, J. Yu, J. Ran, B. Zhang, H. Yan and J. R. Gong, J. Am. Chem. Soc., 2011, 133, 10878-10884.

5 X. Zhang, T. Peng and S. Song, J. Mater. Chem. A, 2016, 4, 2365-2402.

6 X. Chen, S. Shen, L. Guo and S. S. Mao, Chem. Rev., 2010, 110, 6503-6570. 
7 C. Zhao, Z. Chen, R. Shi, X. Yang and T. Zhang, Adv. Mater., 2020, 32, e1907296.

8 G. Zhang, Z. A. Lan and X. Wang, Angew. Chem., Int. Ed., 2016, 55, 15712-15727.

9 A. M. Elewa, M. H. Elsayed, A. F. M. El-Mahdy, C.-L. Chang, L.-Y. Ting, W.-C. Lin, C.-Y. Lu and H.-H. Chou, Appl. Catal., $B, 2021,285,119802$.

10 X. Wang, L. Chen, S. Y. Chong, M. A. Little, Y. Wu, W. H. Zhu, R. Clowes, Y. Yan, M. A. Zwijnenburg, R. S. Sprick and A. I. Cooper, Nat. Chem., 2018, 10, 1180-1189.

11 Y. Yamada, T. Miyahigashi, H. Kotani, K. Ohkubo and S. Fukuzumi, J. Am. Chem. Soc., 2011, 133, 16136-16145.

12 M. Luo, W. Yao, C. Huang, Q. Wu and Q. Xu, J. Mater. Chem. A, 2015, 3, 13884-13891.

13 S. Liu, C. Zhang, Y. Sun, Q. Chen, L. He, K. Zhang, J. Zhang, B. Liu and L.-F. Chen, Coord. Chem. Rev., 2020, 413, 213266.

14 Y. Shi, A.-F. Yang, C.-S. Cao and B. Zhao, Coord. Chem. Rev., 2019, 390, 50-75.

15 H. Lv, Y. V. Geletii, C. Zhao, J. W. Vickers, G. Zhu, Z. Luo, J. Song, T. Lian, D. G. Musaev and C. L. Hill, Chem. Soc. Rev., 2012, 41, 7572-7589.

16 M. Zhang, H. Li, J. Zhang, H. Lv and G.-Y. Yang, Chin. J. Catal., 2020, 42, 855-871.

17 T. Cui, L. Qin, F. Fu, X. Xin, H. Li, X. Fang and H. Lv, Inorg. Chem., 2021, 60, 4124-4132.

18 H. Lv, Y. Gao, W. Guo, S. M. Lauinger, Y. Chi, J. Bacsa, K. P. Sullivan, M. Wieliczko, D. G. Musaev and C. L. Hill, Inorg. Chem., 2016, 55, 6750-6758.

19 H. Lv, W. Guo, K. Wu, Z. Chen, J. Bacsa, D. G. Musaev, Y. V. Geletii, S. M. Lauinger, T. Lian and C. L. Hill, J. Am. Chem. Soc., 2014, 136, 14015-14018.

20 L. Qin, C. Zhao, L.-Y. Yao, H. Dou, M. Zhang, J. Xie, T.-C. Weng, H. Lv and G.-Y. Yang, CCS Chem., 2021, 3, 651-663.

21 M. Zheng, Y. Ding, X. Cao, T. Tian and J. Lin, Appl. Catal., B, 2018, 237, 1091-1100.

22 X. Gao, J. Wang, Q. Xue, Y.-Y. Ma and Y. Gao, ACS Appl. Nano Mater., 2021, 4, 2126-2135.

23 F. M. Toma, A. Sartorel, M. Iurlo, M. Carraro, P. Parisse, C. Maccato, S. Rapino, B. R. Gonzalez, H. Amenitsch, T. Da Ros, L. Casalis, A. Goldoni, M. Marcaccio, G. Scorrano, G. Scoles, F. Paolucci, M. Prato and M. Bonchio, Nat. Chem., 2010, 2, 826-831.

24 B. Chakraborty, G. Gan-Or, M. Raula, E. Gadot and I. A. Weinstock, Nat. Commun., 2018, 9, 4896.

25 S. Pourbeyram, M. Moosavifar and V. Hasanzadeh, J. Electroanal. Chem., 2014, 714-715, 19-24.

26 P. Wang, L. Jiang, X. Zou, H. Tan, P. Zhang, J. Li, B. Liu and G. Zhu, ACS Appl. Mater. Interfaces, 2020, 12, 25910-25919.

27 H. Ma, B. Liu, B. Li, L. Zhang, Y. G. Li, H. Q. Tan, H. Y. Zang and G. Zhu, J. Am. Chem. Soc., 2016, 138, 5897-5903.

28 C.-Y. Sun, S.-X. Liu, D.-D. Liang, K.-Z. Shao, Y.-H. Ren and Z.-M. Su, J. Am. Chem. Soc., 2009, 131, 1883-1888.

29 G. Paille, M. Gomez-Mingot, C. Roch-Marchal, B. LassalleKaiser, P. Mialane, M. Fontecave, C. Mellot-Draznieks and A. Dolbecq, J. Am. Chem. Soc., 2018, 140, 3613-3618.
30 G. Li, K. Zhang, C. Li, R. Gao, Y. Cheng, L. Hou and Y. Wang, Appl. Catal., B, 2019, 245, 753-759.

31 M. Samaniyan, M. Mirzaei, R. Khajavian, H. EshtiaghHosseini and C. Streb, ACS Catal., 2019, 9, 10174-10191.

32 P. Yang, W. Zhao, A. Shkurenko, Y. Belmabkhout, M. Eddaoudi, X. Dong, H. N. Alshareef and N. M. Khashab, J. Am. Chem. Soc., 2019, 141, 1847-1851.

33 X. H. Li, Y. W. Liu, Y. Lu, Z. Zhang, H. R. Tian, S. M. Liu and S. X. Liu, Chem. Commun., 2020, 56, 1641-1644.

34 L. Zeng, X. Guo, C. He and C. Duan, ACS Catal., 2016, 6, 7935-7947.

35 X. J. Kong, Z. Lin, Z. M. Zhang, T. Zhang and W. Lin, Angew. Chem., Int. Ed., 2016, 55, 6411-6416.

36 J. Tian, Z. Y. Xu, D. W. Zhang, H. Wang, S. H. Xie, D. W. Xu, Y. H. Ren, H. Wang, Y. Liu and Z. T. Li, Nat. Commun., 2016, 7, 11580.

37 L. Jiao, Y. Dong, X. Xin, L. Qin and H. Lv, Appl. Catal., B, 2021, 291, 120091, DOI: 10.1016/j.apcatb.2021.120091.

38 Z. M. Zhang, T. Zhang, C. Wang, Z. Lin, L. S. Long and W. Lin, J. Am. Chem. Soc., 2015, 137, 3197-3200.

39 H. Li, S. Yao, H.-L. Wu, J.-Y. Qu, Z.-M. Zhang, T.-B. Lu, W. Lin and E.-B. Wang, Appl. Catal., B, 2018, 224, 46-52.

40 D. Shi, R. Zheng, C. S. Liu, D. M. Chen, J. Zhao and M. Du, Inorg. Chem., 2019, 58, 7229-7235.

41 I. Choudhuri and D. G. Truhlar, J. Phys. Chem. C, 2020, 124, 8504-8513.

42 X. Li, K. Gao, B. Mo, J. Tang, J. Wu and H. Hou, Inorg. Chem., 2021, 60, 1352-1358.

43 A. W. Peters, Z. Li, O. K. Farha and J. T. Hupp, ACS Appl. Mater. Interfaces, 2016, 8, 20675-20681.

44 C. T. Buru, M. C. Wasson and O. K. Farha, ACS Appl. Nano Mater., 2019, 3, 658-664.

45 C. T. Buru, P. Li, B. L. Mehdi, A. Dohnalkova, A. E. PlateroPrats, N. D. Browning, K. W. Chapman, J. T. Hupp and O. K. Farha, Chem. Mater., 2017, 29, 5174-5181.

46 S. Cao, J. Jiang, B. Zhu and J. Yu, Phys. Chem. Chem. Phys., 2016, 18, 19457-19463.

47 J. F. Guayaquil-Sosa, B. Serrano-Rosales, P. J. Valadés-Pelayo and H. de Lasa, Appl. Catal., B, 2017, 211, 337-348.

48 P. J. Kulesza, M. Chojak, K. Karnicka, K. Miecznikowski, B. Palys, A. Lewera and A. Wieckowski, Chem. Mater., 2004, 16, 4128-4134.

49 Y. Liu, Y. Li, F. Peng, Y. Lin, S. Yang, S. Zhang, H. Wang, Y. Cao and H. Yu, Appl. Catal., B, 2019, 241, 236-245.

50 M. Luo, P. Lu, W. Yao, C. Huang, Q. Xu, Q. Wu, Y. Kuwahara and H. Yamashita, ACS Appl. Mater. Interfaces, 2016, 8, 20667-20674.

51 E. Park, J. Jack, Y. Hu, S. Wan, S. Huang, Y. Jin, P. C. Maness, S. Yazdi, Z. Ren and W. Zhang, Nanoscale, 2020, 12, 25962602.

52 I. Vamvasakis, B. Liu and G. S. Armatas, Adv. Funct. Mater., 2016, 26, 8062-8071.

53 J. He, J. Wang, Y. Chen, J. Zhang, D. Duan, Y. Wang and Z. Yan, Chem. Commun., 2014, 50, 7063-7066.

54 M. Lan, R.-M. Guo, Y. Dou, J. Zhou, A. Zhou and J.-R. Li, Nano Energy, 2017, 33, 238-246. 
55 P. Ling, J. Lei, L. Jia and H. Ju, Chem. Commun., 2016, 52, 1226-1229.

56 Y. Liu, Z. Liu, D. Huang, M. Cheng, G. Zeng, C. Lai, C. Zhang, C. Zhou, W. Wang, D. Jiang, H. Wang and B. Shao, Coord. Chem. Rev., 2019, 388, 63-78.

57 X. Ma, L. Wang, Q. Zhang and H. L. Jiang, Angew. Chem., Int. Ed., 2019, 58, 12175-12179.

58 M. Sun, C. Sun, X. Wang and Z. Su, Catal. Commun., 2020, 137, 105930.

59 C. Wang, K. E. deKrafft and W. Lin, J. Am. Chem. Soc., 2012, 134, 7211-7214.

60 T. Hsu-Yao, K. P. Browne, N. Honesty and Y. J. Tong, Phys. Chem. Chem. Phys., 2011, 13, 7433-7438.

61 U. Lee, H. C. Joo, K. M. Park, S. S. Mal, U. Kortz, B. Keita and L. Nadjo, Angew. Chem., Int. Ed., 2008, 47, 793-796.

62 S. Li, S. Liu, S. Liu, Y. Liu, Q. Tang, Z. Shi, S. Ouyang and J. Ye, J. Am. Chem. Soc., 2012, 134, 19716-19721.

63 B. Zhang, G. Sun, S. Ding, H. Asakura, J. Zhang, P. Sautet and N. Yan, J. Am. Chem. Soc., 2019, 141, 8185-8197.

64 Z. Zhang, Q. Lin, S. T. Zheng, X. Bu and P. Feng, Chem. Commun., 2011, 47, 3918-3920.
65 W. Guo, H. Lv, Z. Chen, K. P. Sullivan, S. M. Lauinger, Y. Chi, J. M. Sumliner, T. Lian and C. L. Hill, J. Mater. Chem. A, 2016, 4, 5952-5957.

66 F. Bozheyev, F. Xi, P. Plate, T. Dittrich, S. Fiechter and K. Ellmer, J. Mater. Chem. A, 2019, 7, 10769-10780.

67 P. P. Bag, X. Wang, P. Sahoo, J. Xiong and R. Cao, Catal. Sci. Technol., 2017, 7, 5113-5119.

68 I.-M. Mbomekalle, Y. W. Lu, B. Keita and L. Nadjo, Inorg. Chem. Commun., 2004, 7, 86-90.

69 J. E. Mondloch, W. Bury, D. Fairen-Jimenez, S. Kwon, E. J. DeMarco, M. H. Weston, A. A. Sarjeant, S. T. Nguyen, P. C. Stair, R. Q. Snurr, O. K. Farha and J. T. Hupp, J. Am. Chem. Soc., 2013, 135, 10294-10297.

70 T. C. Wang, N. A. Vermeulen, I. S. Kim, A. B. Martinson, J. F. Stoddart, J. T. Hupp and O. K. Farha, Nat. Protoc., 2016, 11, 149-162.

71 C. M. Sánchez-Sánchez, J. Solla-Gullón, F. J. Vidal-Iglesias, A. Aldaz, V. Montiel and E. Herrero, J. Am. Chem. Soc., 2010, 132, 5622-5624. 